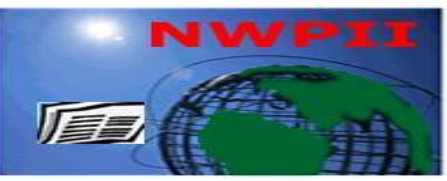

American Journal of Biomedical Sciences

ISSN: 1937-9080

nwpii.com/ajbms

\title{
Could Hesperidin and Iron chelator be a new Therapeutic approach in Gentamicin induced Acute Kidney Injury in Rats?
}

\author{
Marwa H. Muhammad \\ Physiology Department, Benha Faculty of Medicine, Benha University, Qalubiya, Egypt \\ "Corresponding Author \\ Marwa H. Muhammad \\ Physiology Department \\ Benha Faculty of Medicine \\ Benha University \\ Qalubiya \\ Egypt \\ Cell no.002 01009655692 \\ Email: drmarwa.hassan@yahoo.com
}

Received: 26 January 2017; | Revised: 31 March 2017; | Accepted: 19 April 2017

\begin{abstract}
Kidney function is maintaining of homeostasis and toxins elimination. Thus, it is susceptible to acute damage by xenobiotics. Drug-induced nephrotoxicity is becoming a principal etiology for acute kidney injury (AKI). The present study was designed to investigate the protective effect of iron chelator, desferroxamin (DFO), and hesperidin (HES) on gentamicin (GM) induced AKI in rats and elucidating the potential mechanisms. Group I: control, group II: GM (100mg/kg/d. i.p.) for 7days, group III: HES (200 mg/ $\mathrm{kg}$ ) two days before and 7 days concomitantly with GM, group IV: DFO (100 mg/kg/d. i.p.) coadministrated with GM and group V: GM + combined treatment by DFO and HES. Serum urea, creatinine, KIM1, TNF- $\alpha$ and IL-6, renal MDA and HO1 concentration, immunohistochemical expression of caspase 3 and histopathology of kidney were evaluated. GM induced significant increase in all parameters excluding renal HO1 level which is significantly decreased $(\mathrm{p}<0.05)$. Histopathological examinations revealed acute tubular necrosis, hemorrhage and interstitial nephritis. These results were significantly reversed upon either therapy by DFO or HES with more protection by combined treatment. These findings suggest that the Renoprotective mechanisms of HES and DFO including antioxidant, anti-inflammatory and anti-apoptotic effects. They are a good therapeutic approach for treating gentamicin nephrotoxicity.
\end{abstract}

Keywords: Desferroxamin, Hesperidin, Acute kidney injury, Gentamicin

Abbreviations: (AKI): Acute kidney injury; (GM): Gentamicin; (DFO):Desferroxamin; (HES):Hesperidin; (KIM1): Kidney injury molecule 1;(HO1): Heamoxygenase 1 


\section{Introduction}

Acute kidney injury (AKI) is a major kidney disease characterized by rapid loss of renal function. This leads to accumulation of metabolic waste products and disturbed body water and electrolytes balance [1]. The rising incidence of AKI in both creating and created nations turned it into a worldwide general wellbeing concern associated with high morbidity, mortality, and health services cost [2]. The overall mortality after AKI remains high and is around half [3].

Drug-induced AKI is estimated by about $22.2 \%$ of the 100 most used drugs in intensive care unit were nephrotoxic [4].A prominent cause of drug-induced AKI is aminoglycosides. They are antibiotics used in clinical settings to treat serious Gram-negative bacterial infections. Nephrotoxic complication due to their use occurs in $10-20 \%$ of therapeutic regimes [1].

Gentamicin (GM), one of aminoglycosides, is recommended as the first line antibiotic for many and life-threatening diseases because of its clinical effectiveness, a low rate of resistance and reasonable cost [5].Most studies report gentamicin nephrotoxicity rates of between $8 \%$ and $26 \%$ [6]. No helpful medications improve survival, restrain damage or fasten recovery from gentamicin induced AKI other than dialysis. Several planes of treatment have been used, with a variety of impact, trying to invert the gentamicin promoted AKI [2].Therefore, there is a pressing need to find a new therapeutic strategy to treat AKI.

Hesperidin (HES) is a standout amongst the most important bioflavonoid. It is dominating in citrus fruits especially in the peel and membranous parts of sweet orange and lemon [7]. It is likewise in vegetables, nuts and seeds. Daflon, a dietary supplement, is also a source for hesperidin [8].Hesperidin is a strong antioxidant and one of naturally occurring iron chelators [9]. In addition, it suppresses inflammation [10].

Desferoxamine (DFO) has been the standard for iron-chelation therapy. It is used for treatment of iron- overload syndromes. There is a rising role for iron chelators in the treatment of non-iron overload diseases [11]. For the association between renal diseases and iron, a study stated that angiotensin II increased renal iron deposition and altered the expression of renal iron transporters in rats [12].Also, progression of diabetic nephropathy is affected by a low-iron diet [13].These findings give evidence that iron is involved in the pathology of renal damage and iron reduction could ameliorate kidney diseases. However, the effect of iron reduction on AKI induced by GM stays indistinct.

Our strategy relies on treatment with possible renoprotective agents to alleviate the GM induced AKI based on specific mechanisms of action. We investigated the possibility of oxidative stress, inflammation and apoptosis in its pathogenesis and the potential role of HES and DFO therapy.

\section{Materials and Methods}

\subsection{Experimental Animals}

Adult male albino rats weighing 230-260 g were used in the present study. They were obtained from the animal research center, faculty of veterinary medicine, Benha University, Egypt. The animals were housed (4 rats per cage) and maintained on 12:12 $\mathrm{h}$ day and night cycles at controlled temperature. The animals were fed with standard pellet diets and drinking water ad libitum. They were subjected to an adaptation period of 2 weeks before experiments. This study was carried out in strict accordance with the recommendations in the Guide for the Care and Use of Laboratory Animals of the National Institutes of Health. The protocol was approved by the Committee on the Ethics of Animal Experiments of the Faculty of Medicine, Benha University. Anesthesia by sodium pentobarbital $(50 \mathrm{mg} / \mathrm{kg}$, intra-peritoneal) was performed, and all efforts were made to minimize suffering.

\subsection{Chemicals and reagents}

Gentamicin and Desferroxamin were purchased from local market E.P.I.C.O. and Novartis, respectively (Egypt). Hesperidin was provided by Sigma Chemical Co (St. Louis, MO, USA). The TNF- $\alpha$, IL6, serum KIM-1 [TIM-1 Rat ELISA Kits] and malondialdehyde, MDA, TBARS assay kits were acquired from Abcam (Cambridge, UK). Rat specific, heamoxygenase-1 enzyme-linked immunosorbent assay kit [HO-1, rat, EIA. ADIEKS-810A] was obtained from Enzo Life Sciences International, Inc. (New York, USA). Primary 
antibodies for Caspase 3 were acquired from Santa Cruz Biotech (Dallas, TX, USA). Goat anti-mouse peroxidase-conjugated secondary antibodies were from DakoCytomation (Glostrup, Denmark). Urea and creatinine assay kits were obtained from Diamond Diagnostics Company (Egypt).

\subsection{Experimental design}

Forty adult albino rats were randomly divided into five equal groups (8 rats per group). Both HES and DFO were dissolved in saline $(0.9 \% \mathrm{NaCl})$ and the solutions were prepared immediately before use. Group I (Control): rats were administered saline by intra-peritoneal injection (i.p.) throughout the experimental period. Group II (gentamicin group): rats were injected (i.p.) with gentamicin at a dose of $(100 \mathrm{mg} / \mathrm{kg} /$ day $)$ for seven days and served as gentamicin control. GM injections were carried out between 09.00 and $09.30 \mathrm{~h}$ to maximize GMprovoked nephrotoxicity as circadian variation in GM-induced nephrotoxicity is well known [14]. Group III (HES+GM): rats were treated with hesperidin by oral gavage at dose $200 \mathrm{mg} / \mathrm{kg}$ two days before and seven days concomitantly with gentamicin $(100 \mathrm{mg} / \mathrm{kg} / \mathrm{d}$, i.p.). The hesperidin dose was in according with [15]. Group IV (DFO+GM): rats were treated with DFO $(100 \mathrm{mg} / \mathrm{kg} / \mathrm{d}$, i.p. $)$ for seven days in concomitantly with gentamicin (100 $\mathrm{mg} / \mathrm{kg} / \mathrm{d}$, i.p.). The DFO dose used in this study was chosen from a previous study by [16] and from another one by [17]. Group V (HES+DFO+GM): rats in this group were treated orally with hesperidin at dose $200 \mathrm{mg} / \mathrm{kg}$ two days before and seven days concomitantly with gentamicin $(100 \mathrm{mg} / \mathrm{kg} / \mathrm{d}$, i.p. $)$ and DFO (100 mg/kg/d, i.p.).

At the end of experiment, the animals were anesthetized with sodium pentobarbital $(60 \mathrm{mg} / \mathrm{kg}$ i.p.), then the blood sample was obtained by cardiac puncture from the right ventricle. The blood was left until clotting. Serum was separated by centrifugation at 3000 revolution per minute for 15 min and stored at $-80^{\circ} \mathrm{C}$. The kidneys were exposed then freed from the surrounding tissues. One kidney was immediately kept in $10 \%$ neutralized formalin for morphological analysis. The other kidney was divided into two longitudinal sections. Renal tissue was isolated and kept. It then homogenized in apposite buffer in cold condition $(\mathrm{pH} \mathrm{7.0)}$ to give $20 \%$ homogenate (w/v). The homogenate was centrifuged at $1000 \mathrm{rpm}$ for $10 \mathrm{~min}$ at $4{ }^{\circ} \mathrm{C}$. The supernatant was used for various analyses.

\subsection{Laboratory analysis}

a. Measurement of serum urea and creatinine

Estimation of Serum Blood Urea Nitrogen (BUN) Level and creatinine were by enzymatic colorimetric methods using commercial kits as described by [18]. The data were expressed as $\mathrm{mg} / \mathrm{dL}$.

\section{b. Measurement of serum kidney injury} molecule-1(KIM-1)

It is measured by using kidney injury molecule-1, KIM-1/ TIM-1/ rat ELISA kit which is an in vitro enzyme-linked Immuno-sorbent assay for the quantitative measurement of samples in serum [19].

\section{c. Measurement of renal lipid peroxidation MDA}

In order to determine conditions of renal oxidative stress, the measurement of malondialdehyde (MDA) level, a marker of lipid peroxidation, in the kidney was carried out. The supernatants of renal tissue homogenate were collected for the determination of MDA concentration using a commercial TBARS assay kit, as previously described [20].The absorbance values were measured using a microplate reader and were expressed as total MDA level to total protein concentration (nmol/mg protein).

\section{d. Enzyme-Linked Immunosorbent Assay for Hemeoxygenase- 1}

Renal HO-1, a heam degrading enzyme with antioxidant properties, was detected in renal tissue homogenate. It was quantified using commercially available, rat specific, enzyme-linked immunosorbent assay kit. All samples were assayed in duplicate according to the manufacturer's instructions. The intensity of the color was measured in a microplate reader at $450 \mathrm{~nm}$. Sample HO-1 concentrations were determined by interpolating absorbance readings from a standard curve generated with the calibrated $\mathrm{HO}-1$ protein standard. Data was expressed as $\mu \mathrm{g} / \mathrm{g}$ renal tissue [21].

e. Measurement of serum Tumor necrosis factor $\alpha$ (TNF- $\alpha$ ) and Interleukin-6 (IL-6)

Serum levels of TNF $\alpha$ and IL6 were assessed by specific rat ELISA kits. According to the manufacturer's instructions the concentrations of 
both were determined spectrophotometrically at 450 nm. Standard plot was constructed by using standard cytokine and the concentrations for unknown samples were calculated from the standard plot.

\subsection{Immunohistochemical analysis of Caspase 3}

Caspase-3 technique was used for apoptosis detection. The following steps were done according to the manufacturer's instructions. Deparaffinization of formalin-fixed paraffin sections of the kidney was occurred in xylene. Then sections were rehydrated in ascending grades of ethanol. Endogenous peroxidase activity was quenched with peroxidase block for $5 \mathrm{~min}$. Phosphate-buffered saline (PBS) was used to wash sections twice. The slides were incubated with the caspase- 3 primary antibody in a dilution of 1:100 overnight at $4{ }^{\circ} \mathrm{C}$. Sections were then washed twice with PBS. Application of Goat anti-mouse peroxidase-conjugated secondary antibody at a dilution of 1:200 to sections was carried out and incubated for $30 \mathrm{~min}$ at room temperature. It was then followed by washing in distilled water and counterstaining with haematoxylin. Sections were assessed using light microscopy(Olympus Corporation, Tokyo, Japan).

\subsection{Histo-pathological analysis}

Table 1.Renal biochemical markers in control and experimental groups

\begin{tabular}{lccc}
\hline & Serum urea (mg/dl) & Creatinine(mg/dl) & Serum KIM 1 (Pg/mL) \\
\hline Group I [Control] & $11.11 \pm 1.87$ & $0.59 \pm .04$ & $205.83 \pm 21.65$ \\
Group II [GM] & $39.15 \pm 3.79 \llbracket$ & $2.13 \pm .24$ II & $664.83 \pm 49.39 \mathbb{I}$ \\
Group III [HES+GM] & $26.25 \pm 2.13 \S \mathbb{I}$ & $1.4 \pm .19 \S \mathbb{I}$ & $450.17 \pm 40.84 \S \mathbb{I}$ \\
Group IV [DFO+GM] & $24.56 \pm 1.12 \S \mathbb{I}$ & $1.56 \pm .31 \S \mathbb{I}$ & $454.33 \pm 38.03 \S \mathbb{I}$ \\
Group V [HES+DFO+GM] & $12.21 \pm 1.78 \S$ & $0.62 \pm .09 \S$ & $220.50 \pm 14.24 \S$
\end{tabular}

Values are mean \pm SE; ( $n=8$ rats in each group). GM, gentamicin; HES, hesperidin; DFO, desferroxamin; KIM 1, kidney injury molecule-1;

II $(\mathrm{P}<.05)$ compared with control group;

$\S(\mathrm{P}<.05)$ compared with gentamicin group. 
Table 2.Oxidative stress and inflammatory markers in control and experimental groups

\begin{tabular}{|c|c|c|c|c|}
\hline & $\begin{array}{c}\text { Renal MDA } \\
\text { (nmol/mg) }\end{array}$ & $\begin{array}{c}\text { Renal HO1 } \\
(\mu \mathrm{g} / \mathrm{g})\end{array}$ & $\begin{array}{c}\text { Serum TNFa } \\
(\mathrm{Pg} / \mathrm{mL})\end{array}$ & $\begin{array}{c}\text { Serum IL6 } \\
(\mathrm{Pg} / \mathrm{mL})\end{array}$ \\
\hline Group I [Control] & $1.62 \pm .26$ & $1.63 \pm .11$ & $12.11 \pm 1.04$ & $114.13 \pm 1.57$ \\
\hline Group II [GM] & $2.65 \pm .24$ II & $0.81 \pm .13$ II & $29.32 \pm 2.14 \mathrm{I}$ & $270.50 \pm 8.36 \mathbb{I}$ \\
\hline Group III [HES+GM] & $2.21 \pm .18 \S \mathbb{~ I}$ & $1.31 \pm .08 \S \mathbb{~ I ~}$ & $18.18 \pm 1.34 \S \rrbracket$ & $197.81 \pm 1.88 \S \mathbb{I}$ \\
\hline Group IV [DFO+GM] & $2.19 \pm .27 \S \mathbb{I}$ & $1.35 \pm .01 \S \mathbb{I}$ & $18.23 \pm 1.53 \S \Phi$ & $200.68 \pm 2.84 \S \rrbracket$ \\
\hline Group V[HES+DFO+GM] & $1.40 \pm .3 \S$ & $1.62 \pm .09 \S$ & $12.72 \pm .65 \S$ & $118.70 \pm 5.11 \S$ \\
\hline
\end{tabular}

Values are mean \pm SE; $(n=8$ rats in each group). GM, gentamicin; HES, hesperidin; DFO, desferroxamin; HO-1, Hemeoxygenase-1;

II $(\mathrm{P}<.05)$ compared with control group;

$\S(\mathrm{P}<.05)$ compared with gentamicin group.

\subsection{Effect of hesperidin on gentamicin induced acute kidney injury}

By administration of hesperidin to gentamicin treated rats there was a significant decrease in serum urea, creatinine, KIM1, TNF $\alpha$, IL6 and renal tissue MDA levels. There wassignificant increase in renal HO1 concentration compared to gentamicin group ( $\mathrm{p}<0.05)$, asshown in Table 1 and 2.

\subsection{Effect of desferoxamine on gentamicin induced acute nephrotoxicity}

By administration of desferoxamine to gentamicin treated rats there was a significant decrease in serum urea, creatinine, KIM1, TNF $\alpha$, IL6and renal MDA levels. A significant increase in renal HO1 concentration was found when compared to gentamicin group $(\mathrm{p}<0.05)$, Table 1 and 2 .

\subsection{Effect of combination of hesperidin and desferoxamine on gentamicin induced acute nephrotoxicity}

As shown in table 1 and 2, by administration of both hesperidin and desferoxamine to gentamicin treated rats there were a significant decrease in serum urea, creatinine, KIM1, TNF $\alpha$, IL6 and renal tissue MDA levels and a significant increase in renal HO1 concentration when compared to gentamicin group $(\mathrm{p}<0.05)$.

\subsection{The effect of hesperidin and desferroxamin on apoptotic protein caspase- 3 expression in the gentamicin-induced AKI}

The immunohistochemical expression of caspase-3 apoptotic protein was presented in figure 1. Photomicrograph of kidney sections from control group showing no expression of brownish immunereactive caspase-3 cells (Fig.1A) while, that of gentamicin group showed strong positive brownish caspase-3 reaction in all renal tubules (Fig.1B). Photomicrographs of kidney sections from hesperidin + gentamicin group (Fig.1C) and desferroxamin + gentamicin group (Fig.1D) showed weak positive brownish caspase- 3 reaction in few renal tubules. Combined hesperidin and desferroxamin therapy reversed the effect gentamicin by reducing the expression level of caspase-3 (Fig. 1E).

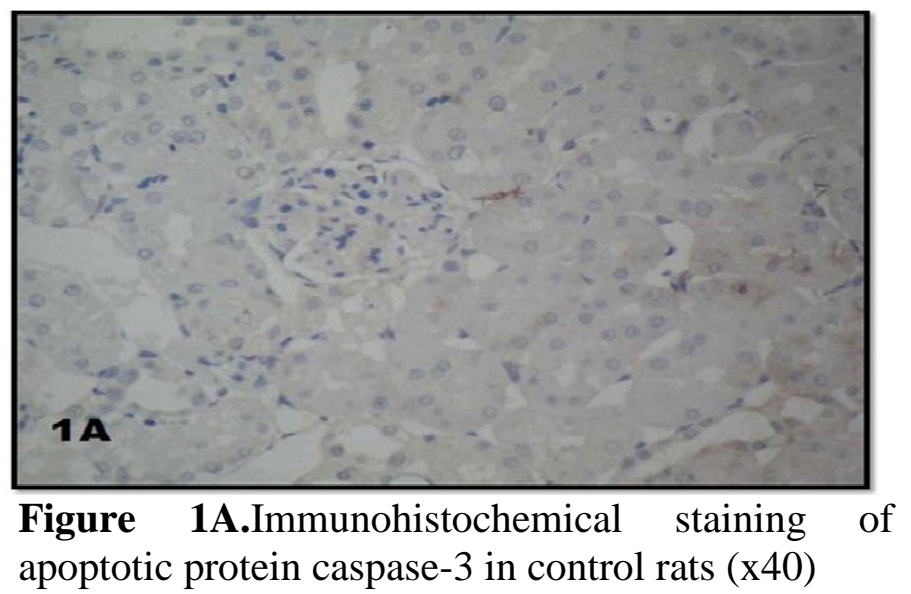




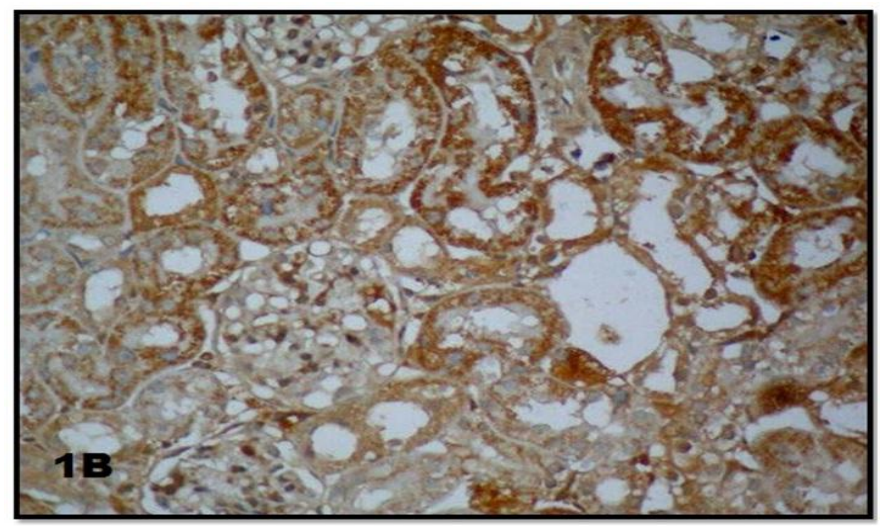

Figure 1B.Caspase-3 immunohistochemical staining in gentamicin group. It revealed a marked increase in the number of positive brownish caspase-3 immune-reactive cells in the kidney (x40)

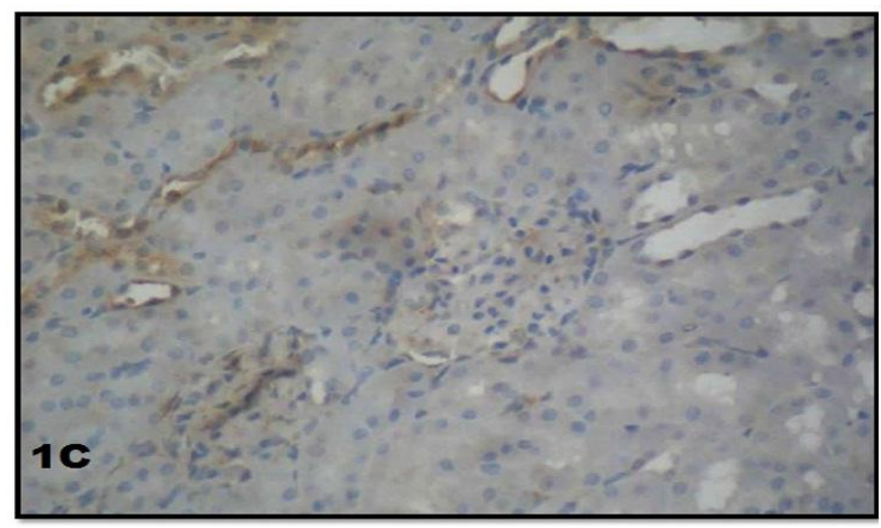

Figure 1C.Caspase-3 expression in gentamicin+ hesperidin group. It shows weak positive brownish caspase- 3 reaction in few renal tubules $(\mathrm{x} 40)$

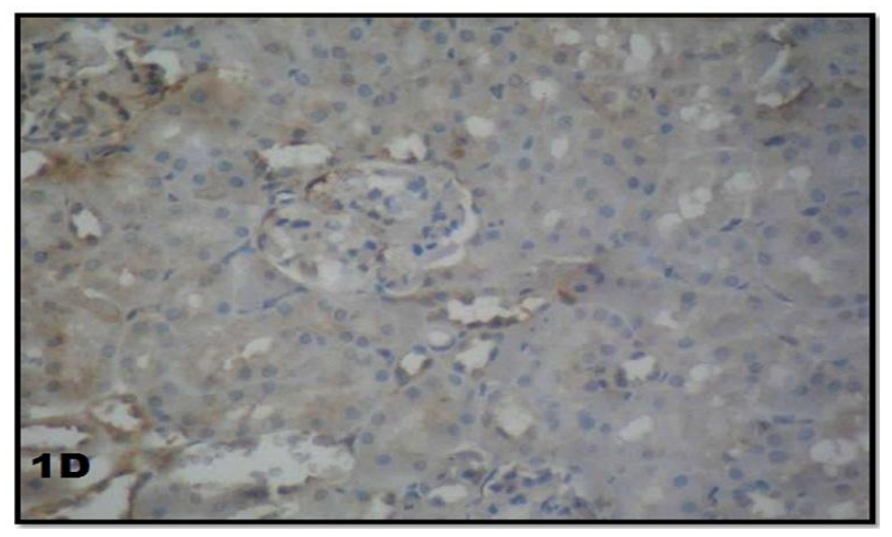

Figure 1D.Immunohistochemical staining of caspase-3 in gentamicin+ desferroxamin group. It shows weak positive brownish caspase- 3 reaction in few renal tubules $(\mathrm{x} 40)$

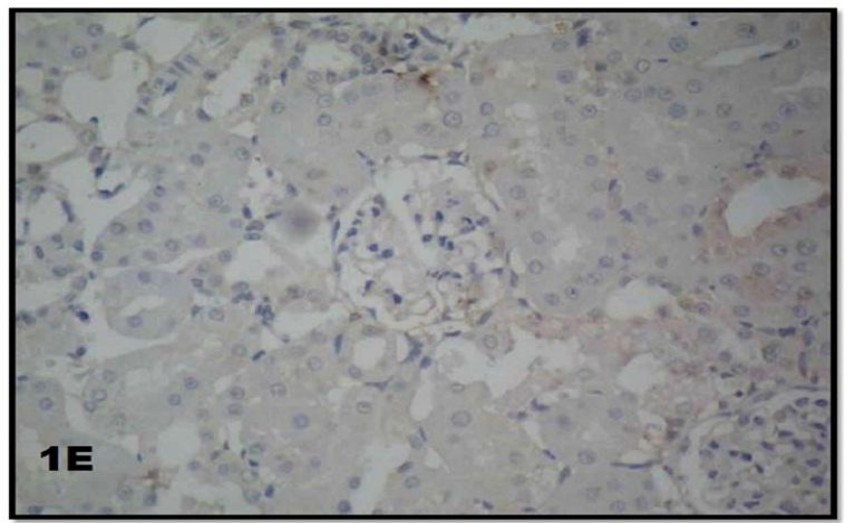

Figure 1E. Immunohistochemical staining of caspase-3 in gentamicin+ hesperidin+ desferroxamin group. It shows marked decrease in caspase- 3 expression in ( $\mathrm{x} 40)$

\subsection{The effect of hesperidin and desferroxamin on renal morphology in the gentamicin-induced AKI}

The histopathological manifestations of the renal tissue are presented in figure 2. Normal kidney morphology was observed in the control group (Fig.2A). Nephrotoxicity in the gentamicintreated rats was evidenced by massive tubular necrosis, interstitial hemorrhage, intra-luminal cast and interstitial nephritis with lymphocytic infiltration (Fig.2B- a \& b). However, these changes were reversed by the combined hesperidin and desferroxamin treatment with gentamicin group (Fig.2E). Hesperidin plus gentamicin group (Fig.2C) and desferroxamin plus gentamicin group (Fig.2D) ameliorated the extent of histological injury and reduced inflammatory infiltration in renal tissue with preserved renal architectures.

\section{Discussion}

A sudden decrease in renal excretory function is defined as acute kidney injury [22].Gentamicin induces acute renal damage in $25 \%$ of treated patients and even more in risky people [23]. Therefore, an in-depth understanding of gentamicininduced kidney damage mechanisms and potential protective agents is essential.

In the present study; GM treated rats exhibited significant increase in serum urea, creatinine and KIM-1 levels versus control group $(\mathrm{P}<0.05)$ Table 1. KIM-1 is a biomarker of renal diseases entailing 


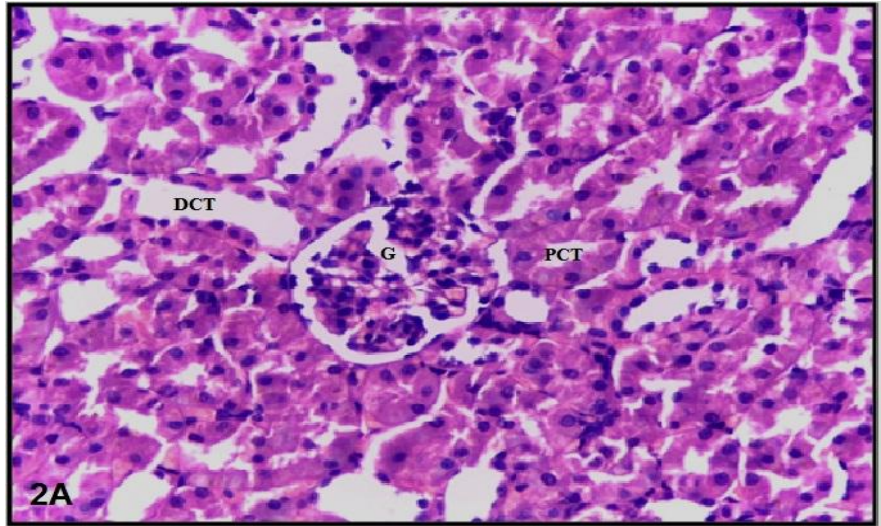

Figure 2A.Histopathological examination of control rats by $H \& E$ stain. It shows normal renal structure [glomerulus, G; proximal convoluted tubules, PCT; distal convoluted tubules, DCT] (x40)

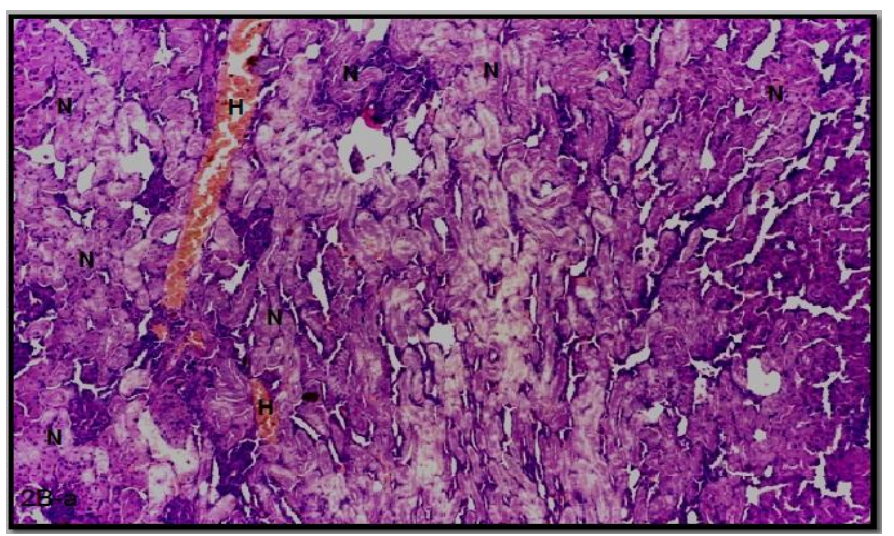

Figure (2B-a).Effect of gentamicin treatment on rat kidney. It shows massive tubular necrosis $[\mathrm{N}]$ and interstitial hemorrhage $[\mathrm{H}]$ and with loss of renal architectures (x20)

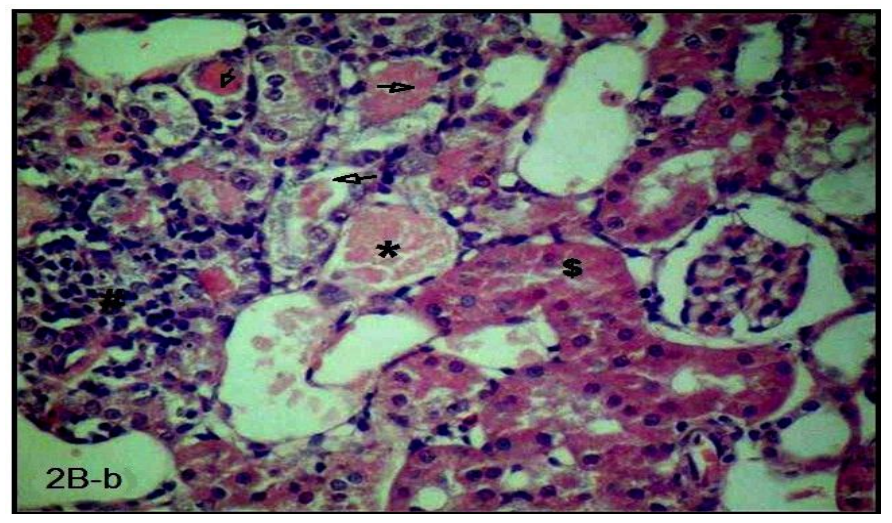

Figure (2B-b). Effect of gentamicin treatment on rat kidney. It showedlymphocytic infiltration [\#], intra tubular cast [black arrows], tubular hyaline degeneration [\$] and congested blood vessel [*] (x40)

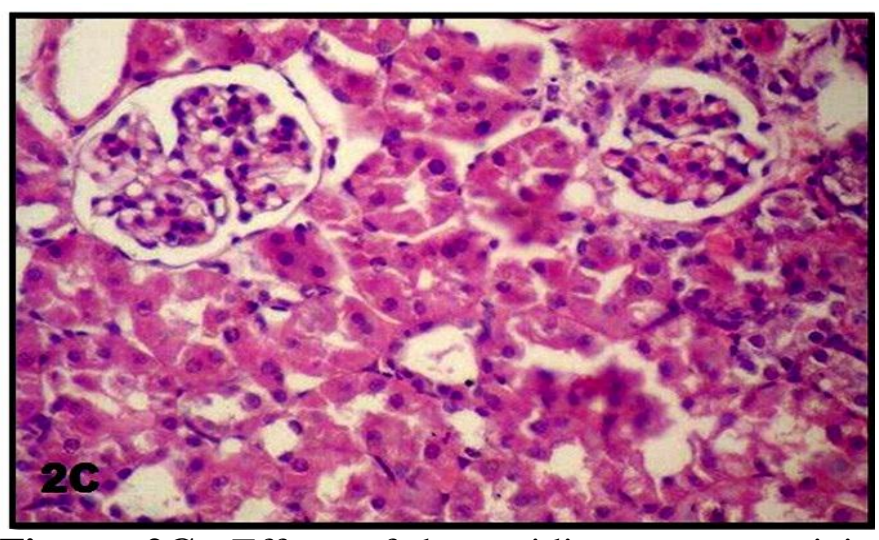

Figure 2C. Effect of hesperidin on gentamicin induced nephrotoxicity in rat kidney showing preserved renal architecture (x40)

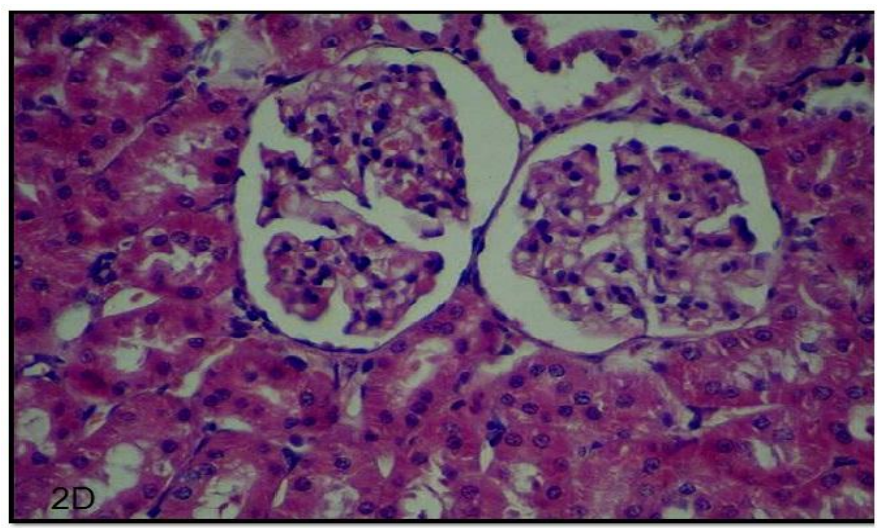

Figure 2D. Effect of desferroxamin on gentamicin induced nephrotoxicity in rat kidney showing preserved renal architecture $(\mathrm{x} 40)$

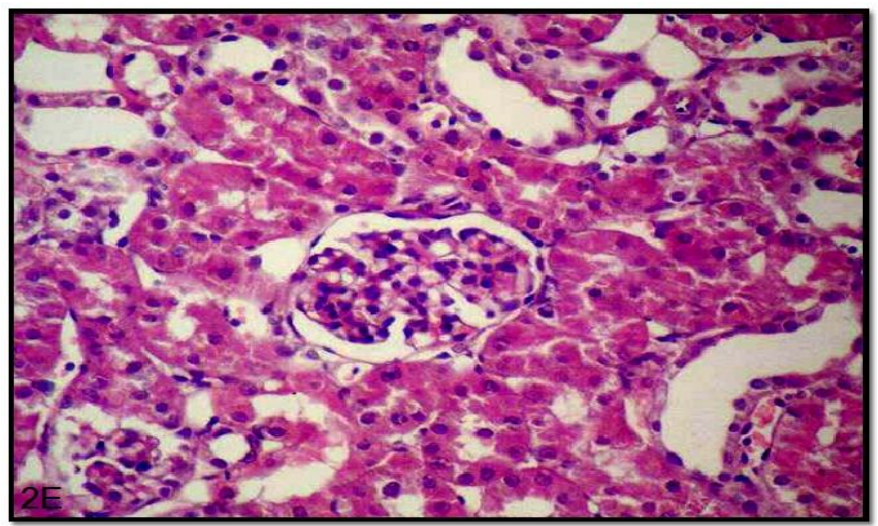

Figure 2E. Effect of combined treatment by hesperidin and desferroxamin on gentamicin induced nephrotoxicity in rat showing nearly pattern of normal renal morphology (x40) 
acute injury of the proximal tubule epithelium [24]. That explains the specificity of GM accumulation in the proximal convoluted tubules [25] and in lysosomes through binding with the phosphatidylinositide receptors [26].This confirming AKI induced by GM.

AKI was associated with significant increase in MDA level when compared to control group. That is in consistent with the finding of [27]. GM induced lipid peroxidation is through superoxide anion (O-), hydrogen peroxide (H2O2), and hydroxyl radical $(\mathrm{OH})$ production from renal mitochondria [28]. It binds to iron forming an irongentamicin complex which significantly promoted free radicals and ROS generation [29]. Free iron also, acts as a catalyst in the chain reactions of lipid peroxidation and membrane dysfunction [30]. This is accompanied with significant $(\mathrm{P}<0.05)$ decrease in renal heamoxygenase $1(\mathrm{HO} 1)$. It is a rate limiting enzyme in heam catabolism, induced by stressors and represents a leading cellular defense mechanism against ROS damage [31]. Its decrease explained by that free radicals generation was followed by depletion of antioxidant enzymes during the process of counteracting oxidative stress [21]. Kidneys are vulnerable to ROS damage due to plenty of long chain polyunsaturated fatty acids in its lipid components [32], this leads to serious oxidative damage and functions deterioration.

Our data revealed significant increase in TNF$\alpha$ and IL6 in gentamicin group when compared to control ( $\mathrm{p}<0.05)$. TNF- $\alpha$ enhances the production of other cytokines interceding inflammation [33] and IL-6 stimulates neutrophils release from the bone marrow and activating them to produce toxic enzymes. This is supported by the histopathological results which revealed the presence of lymphocytic cells infiltration in kidney sections of GM group (Fig. 2B-b). In this regard, ROS activates nuclear factor kappa $\mathrm{B}(\mathrm{NF}-\mathrm{\kappa B})$ that plays a key role in the inception of inflammatory process [34]. Furthermore, we found marked increase in the number of brownish caspase-3 immune-reactive cells in GM group when compared to control (Fig. 1B) indicating involvement of apoptosis in pathogenesis of GM nephrotoxicity. This was in agreement with the results of [35] and explained by that excess of intra-lysosomal iron increases its permeability leading to release of cathepsins into the cytosol. This will induce apoptosis through activation of mitochondrial membrane permeabilization [36].

From our results we suppose that pathogenesis of AKI induced by GM involves oxidative stress, inflammatory reactions and apoptotic cell death. This goes parallel with histopathological findings of massive tubular necrosis, hemorrhage and loss of renal architectures (Fig. 2B-a). Interstitial nephritis, congested blood vessel and intra-luminal hyaline casts (Fig. 2B-b). That was consistent with findings of [37].

Iron-generated ROS plays a role in pathogenesis of gentamicin induced AKI. We supposed that iron chelation and antioxidants may have a therapeutic value in gentamicin nephrotoxicity. For this purpose we compared between the effect of classic iron chelator desferroxamin and hesperidin as potent antioxidant in gentamicin nephrotoxicity. In (DFO + GM) group and (HES + GM) group; we found significant decrease in renal function parameters, serum urea, creatinin and KIM-1, oxidative stress markers, MDA and renal HO-1 concentration, and inflammatory markers, TNF $\alpha$ and IL6 when compared to gentamicin group $(\mathrm{P}<0.05)$ Table 1 and 2. There was decreased caspase-3 expression in kidney sections from these groups when compared to gentamicin group (Fig. 1C and 1D). This means that DFO and HES exerts a renoprotective effect which was further confirmed by histopathological results in which there were improvement of renal architecture with absence of tubular necrosis, hemorrhage, intra-tubular cast or lymphocytes infiltration (Fig. 2C and 2D).

The renoprotective effect of desferoxamine was based on the idea of a "push-and-pull" mechanism to scavenge redox active transition metals. The chelators are able to tightly bind redox active metals preventing them from participation in the ROS production [38]. DFO is endocytosed and accumulates in lysosomes where ROS generated [23]. By the same way, DFO removes iron from the enzymes active site that play key role in various oxidative damage [39]. Furthermore, iron chelators suppress oxidative stress by inhibiting the activity of NADPH oxidase and suppressed expression of its subunit p22 ${ }^{\text {phox }}$ [40]. Additionally, DFO induces HO-1 expression by blocking of p38 signaling 
pathway [41]. The decreased IL6 by DFO was in agreement with Demetrios Vlahakos et al. [42]. They reported increased IL-6 production when endothelial cells incubated with iron and blocked by co-incubation with DFO. Decreased caspase-3 expression by DFO was in agreement with [43]. Being, DFO common lysosomal target iron chelator causes iron starvation and decreased the lysosomal and mitochondrial membrane permeabilization [44].

The renoprotective effect of hesperidin on gentamicin induced AKI was explained by, in addition to its free-radical scavenging activity and restoration of the antioxidant defense systems it is also, a potent iron chelator at different $\mathrm{pH}$ conditions ( $\mathrm{pH} 6.8$ and 7.5). It forms stable complex with iron. This facilitates protection of membrane lipids against ROS injury [45]. Moreover, hesperidin up-regulates HO-1 expression through increased nuclear factor erythroid 2- related factor (Nrf2) signaling [46].The anti-inflammatory effect of HES has been chiefly associated with its antioxidant activity and ability to inactivate the proinflammatory cascade initiated by free radicals. It inhibits of nuclear factor kappa B (NF- $\kappa \mathrm{B})$ expression that triggers pro-inflammatory genes synthesis [10].

By comparing the effect of (DFO + GM) and (HES + GM) groups, we found no significant changes between the two groups $(\mathrm{P}>0.05)$. So, we concluded that desferroxamin and hesperidin showed more or less similar renoprotective effect against GM-induced AKI.

When both desferroxamin and hesperidin were administered with GM, they efficiently protected the rat kidneys from the serious nephrotoxic effects of GM. This is evidenced by comparing the effect of $\mathrm{DFO}+\mathrm{GM}$ group or $\mathrm{HES}+\mathrm{GM}$ group to $\mathrm{HES}+\mathrm{DFO}+\mathrm{GM}$ group we found significant change in all parameters $(p<0.05)$. Also, there were more reduction in caspase-3 expression in this group (Fig.1E) and improvement of renal architectures with absence of histopathological findings of nephrotoxicity (Fig.2E).

These findings can be explained via increased HO- 1 concentration is mediated by two signaling pathways through p38 blocking by DFO and Nrf2 activation by HES. The cellular protective mechanism is exerted by HO-1 itself and by its products $\mathrm{CO}$, iron and biliverdin that converted into bilirubin [47]. Bilirubin is a chain breaking antioxidant that acts as a lipid peroxyl radical (ROO·) scavenger. It also, inhibits NADPH oxidase activity and prevents neutrophils adhesion [48].CO, the next product of heam metabolism, is a tissue protective through its vasodilator, anti-platelet, and direct anti-inflammatory effects [49]. The HO-1 activity increased the expression of ferritin and ferritin synthesis, which serves to sequester iron thereby protecting cells against metal ion, induced oxidative stress [50].

It therefore, appears that combined treatment by both HES and DFO has potential as a promising and multipurpose therapy against gentamicin induced AKI.

\section{Conclusion}

Based on the results of this study, we propose that either hesperidin or desferroxamin could be therapeutically beneficial in the treatment of gentamicin induced AKI, with greater effect of their combination, as a consequence of their antioxidative, anti-inflammatory and anti-apoptotic actions. The iron chelating property, the increased HO-1 level, reduced TNF- $\alpha$ and IL6 alongside inhibition of caspase-3 activity were among the mechanisms that may have contributed to this protection.

\section{Conflict of interest}

The author has declared that no conflict of interest exists.

\section{Funding}

This research received no specific Grant from any funding agency in the public, commercial or not-for-profit sectors.

\section{Acknowledgment}

This research was officially supported by the Physiology Department, Faculty of Medicine, Benha University. Moreover, Author would like to thank Department of Pathology, Faculty of Medicine, Benha University for their co-operation 
in the histopathological analysis of the present work.

\section{References}

[1] Jing Cui, Xue-Yuan Bai, Xuefeng Sun, GuangyanCai, Quan Hong, Rui Ding and Xiangmei Chen (2015); "Rapamycin protects against gentamicin-induced acute kidney injury via autophagy in mini-pig models" Scientific Reports 5:11256 DOI: $10.1038 /$ srep11256.

[2] Zuk, A., and Bonventre, J. V. (2016); "Acute Kidney Injury" Annual Review of Medicine Vol. 67: 293-307. DOI: 10.1146/annurev-med050214-013407.

[3] Siew ED and Davenport A, (2015);"The growth of acute kidney injury: a rising tide or just closer attention to detail?". Kidney International (Review) 87 (1): 46-61.

DOI:10.1038/ki.2014.293

[4] Taber SS and Mueller BA. (2006);"Drugassociated renal dysfunction."Crit Care Clin 22: 357-374. DOI:10.1016/j.ccc.2006.02.003

[5] Stojiljkovic N, Stoiljkovic M, Mihailovic D, Randjelovic P, Ilic S, Gocmanac-Ignjatovic M, Veljkovic M.(2012): "Beneficial Effects of Calcium Oral Coadministration in GentamicinInduced Nephrotoxicity in Rats". Ren Fail Vol. 34, No. 5 , Pages 622-627.

DOI:10.3109/0886022X.2012.664809

[6]Nicholas M. Selby, Susan Shaw, Nicholas Woodier, Richard J. Fluck, Nitin V. Kolhe (2009); "Gentamicin-associated acute kidney injury.’QJM 2009; 102 (12): 873-880.

DOI: 10.1093/qimed/hcp143 PMid:19820138

[7] Russo M., Bonaccorsi, I., Inferrera, V., Dugo, P., and Mondello, L. (2015); "Underestimated sources of flavonoids, limnoids and dietary fiber: Availability in orange's by-products." Journal of Functional Foods, 12, 150-157. http://dx.doi.org/10.1016/j.jff.2014.04.004

[8] VesnaKunti, JasminaBrbori, IvankaHolclajtnerAntunovi and SnežanaUskokovi-Markovi (2014); "Evaluating the bioactive effects of flavonoid hesperidin - A new literature data survey VojnosanitPregl ; 71(1): 60-65. PMID: 24516992 PMid:24516992
[9] Kell DB (2009) Iron behaving badly: inappropriate iron chelation as a major contributor to the aetiology of vascular and other progressive inflammatory and degenerative diseases. BMC Med Genomics. 8 :2. DOI: $10.1186 / 1755-8794-2-2$

[10] Subramanian Perumal, RamaswamyAnandan , Jaime Jacqueline Jayapalan and Onn Haji Hashim (2015); "Hesperidin protects gentamicin-induced nephrotoxicity via Nrf2/HO-1 signaling and inhibits inflammation mediated by NF- $\mathrm{BB}$ in rats" journal of functional foods 13 ( 2015 ) 89-99.

http://doi.org/10.1016/j.jff.2014.12.035

[11] Heather C Hatcher, Ravi N Singh,Frank M Torti and Suzy V Torti(2009); "Synthetic and natural iron chelators: therapeutic potential and clinical use." Future Med Chem.; 1(9): 10.4155/fmc.09.121.

DOI: $10.4155 / \mathrm{fmc} .09 .121$.

[12] Ishizaka N, Saito K, Furuta K, Matsuzaki G, Koike K, et al. (2007); "Angiotensin IIinduced regulation of the expression and localization of iron metabolism-related genes in the rat kidney". Hypertens Res 30: 195-202. DOI:10.1291/hypres.30.195

[13] Ikeda Y, Enomoto H, Tajima S, IzawaIshizawa Y, Kihira Y, et al. (2013); "Dietary iron restriction inhibits progression of diabetic nephropathy in $\mathrm{db} / \mathrm{db}$ mice". Am J Physiol Renal Physiol 304: F1028-1036. DOI:10.1152/ajprenal.00473.2012 PMid:23389454

[14] Pariat, C., Courtois, P., Cambar, J., Piriou, A., \& Bouquet, S. (1988); "Circadian variations in the renal toxicity of gentamicin in rats." Toxicology Letters, 40, 175-182. PMID:2893472

[15] Jain DP and Somani RS (2015); “Antioxidant Potential of Hesperidin Protects Gentamicin Induced Nephrotoxicity in Experimental Rats" Austin J PharmacolTher. 2015; 3(2).1071. austinpublishinggroup.com/pharmacologytherapeutics/download.php?file.../ajpt...

[16] Fayez, Mahmoud F.A.; El AbouScoud, Samia H.; Yassien, Ahmed I. and El Sheikh, Ayman A.(2005); "Effect of vitamin $\mathrm{E}$ an antioxidant and deferoxamine an Iron chelator on gentamicin induced nephrotoxicity in albino 
rats." Journal of the Egyptian Society of Pharmacology and Experimental Therapeutics [The]; 26(1)2005.

[17] Al -Tonsy Ahmad H., Mahmoud E. Awad, Mohamed El-Said Nasr, Awad Abdulalattif, OmranAlfitory, M.N. Khan (2002); "Effect of deferoxamin on gentamicin induced acute renal failure in albino rats" Mansoura J.Forensic Med. Clin. Toxicol.Vol.XNo.1, Jan 2002.mfmctjournal.com/Doc_resource/2002/Ja nuary/1.pdf

[18]Murray R.L. (1984): Creatinine In: Clinical Chemistry; Theory, Analysis and Correlation, Kaplan, L.A. and A.J. Pesce (Eds.). CV Mosby Co., St. Louis, pp: 1247-1253.

$$
\text { www.sciepub.com/reference/141226 }
$$

[19] Chaturvedi S., Farmer T., and KapkeG.F. (2009):"Assay Validation for KIM-1: human urinary renal dysfunction biomarker. Int $\mathbf{J}$ BiolSci; 5(2): 128-134. PMC2631222

[20] Ohkawa, H., Ohishi, N., and Yagi, K. (1979). Assay for lipid peroxides in animal tissues by thiobarbituric acid reaction.Anal.Biochem. 95(2): 351-358.

https://www.ncbi.nlm.nih.gov/pubmed/36810

[21] Richard A. Zager, Ali C.M. Johnson and Kirsten Becker (2012): Plasma and Urinary Heamoxygenase-1 in AKI. J Am SocNephrol 23: 1048-1057, 2012.

DOI: 10.1681/ASN.2011121147.

[22] Al-SaqladiAWM (2016); 'Acute Kidney Injury: New Definitions and Beyond'. J NephrolTher 6: 234. DOI:10.4172/21610959.1000234.

[23] Denamur S , DonatienneTyteca , Jacqueline Marchand-Brynaert, Françoise Van Bambeke ,Paul M. Tulkens , Pierre J. Courtoy , MariePaule Mingeot-Leclerc (2011); "Role of oxidative stress in lysosomal membrane permeabilization and apoptosis induced by gentamicin, an aminoglycoside antibiotic." Free Radical Biology and Medicine 51(9):1656-65.

DOI: 10.1016/j.freeradbiomed.2011.07.015

[24] Jin, Z. K., Tian, P. X.,Wang, X. Z., Xue,W. J., Ding, X. M., Zheng, J., Ding, C. G., Mao, T. C., Duan,W. L., and Xi, M. (2013); "Kidney injury molecule-1 and osteopontin: New markers for prediction of early kidney transplant rejection'. Molecular Immunology, 54, 457-464.

DOI:10.1016/j.molimm.2013.01.013

[25] Abdel-Raheem, I.T., Abdel-Ghany, A.A., and Mohamed, G.A. (2009); "Protective effect of quercetin against gentamicin-induced nephrotoxicity in rats." Biol. Pharm. Bull. 32:61-67. PMID:19122282.

[26] Zeeni, N., Selmaoui, B., Beauchamp, D., Labrecque, G., and Thibault, (2007); "Dietary protein level alters gentamicin-induced nephrotoxicity in rats." Physiology \&Behaviour, 90, 760-770.

DOI: $10.1016 /$ j.physbeh.2006.12.018

[27] Rasha R Ahmed, Ayman M Mahmoud, Mohamed B Ashour, Amira M Kamel (2015); "Hesperidin protects against diethylnitrosamine-induced nephrotoxicity through modulation of oxidative stress and inflammation.'National Journal of Physiology, Pharmacy and Pharmacology Vol 5 Issue 5. DOI: $10.5455 /$ njppp.2015.5.2907201567

[28] Moreira MA, Nascimento MA, Bozzo TA, Cintra A, da Silva SM, Dalboni MA, Mouro MG and HigaEM.(2014); "Ascorbic acid reduces gentamicin-induced nephrotoxicity in rats through the control of reactive oxygen species." Clinical Nutrition, Volume 33, Issue 2, Pages 296-301.

DOI:10.1016/j.clnu.2013.05.005

[29] Aust SD, Morehouse LA, Thomas CE.(1985) 'Role of metals in oxygen radical reactions. Free RadicBiolMed ; 1 :3-25. PMID:3013969

[30] Antonio Ayala, Mario F. Muñoz, and Sandro Argüelles (2014); "Lipid Peroxidation: Production, Metabolism, and Signaling Mechanisms of Malondialdehyde and 4Hydroxy-2-Nonenal'. Oxidative Medicine and Cellular Longevity. Volume 2014, Article ID 360438, 31 pages DOI:10.1155/2014/360438.

[31] He, X. H., Yan, X. T.,Wang, Y. L.,Wang, C. Y., Zhang, Z. Z., and Zhan J. (2014); "Transduced PEP-1-haemoxygenase-1 fusion protein protects against intestinal ischemia/reperfusion injury." Journal of Surgical Research, 187, 77-84. DOI: $\underline{10.12659 / M S M .893924}$ 
[32] Acharya CR, ThakarHN And Vajpeyee SK.(2013); "A study of oxidative stress in gentamicin induced nephrotoxicity and effect of antioxidant vitamin C in Wistar Rats." Natl J Physiol Pharm Pharmacol; 3:14-20.

DOI: $10.5455 / n j p p p .2013 .3 .14-20$

[33] Benedetti, G., Fredriksson, L., Herpers, B., Meerman, J., Water B. and Graauw, M. (2013); "TNF-alpha-mediated NF-kappaB survival signaling impairment by cisplatin enhances JNK activation allowing synergistic apoptosis of renal proximal tubular cells." Biochemical Pharmacology, 85, 274-286. DOI:10.1016/j.bcp.2012.10.012

[34] Gambhir S, Vyas D, Hollis M, Aekka A and Vyas A.(2015); "Nuclear factor kappa B role in inflammation associated gastrointestinal malignancies." World Journal of Gastroenterology: WJG.; 21(11):3174-3183. DOI: $10.3748 /$ wjg.v21.i11.3174

[35] Sally A. Abuelezza, NevienHendawya and Sara Abdel Gawadb (2016); "Alleviation of renal mitochondrial dysfunction and apoptosis underlies the protective effect of sitagliptin in gentamicin-induced nephrotoxicity." Royal Pharmaceutical Society, Journal of Pharmacy and Pharmacology, 68, pp. 523-532.

DOI:10.1111/jphp.12534

[36] Hsu, Y. H., Chen, T. H., Wu, M. Y., Lin, Y. F., Chen,W. L., Cheng, T. H., and Chen, C. H. (2014); "Protective effects of Zhibai Dihuang Wan on renal tubular cells affected with gentamicin-induced apoptosis." Journal of Ethnopharmacology, 151, 635-642. DOI: 10.1016/j.jep.2013.11.031 PMid:24269250

[37]Sahu Bidya Dhar, Srujana Tatireddy, Meghana Koneru, Roshan M. Borkar, Jerald Mahesh Kumar,MadhusudanaKuncha,Srinivas R., Shyam Sunder R.and Ramakrishna Sistla(2014); "Naringin ameliorates gentamicin-induced nephrotoxicity and associated mitochondrial dysfunction, apoptosis and inflammation in rats: possible mechanism of nephro-protection." Toxicol Appl Pharmacol 2014; 277: 8-20.

DOI:10.1016/j.taap.2014.02.022

[38] HaimBibi, VladimirVinokur, DanWaisman, YigalElenberg, AmirLandesberg , Anna Faingersh , Moran Yadid, Vera Brod,
JimyPesin, Eduard Berenshtein, Ron Eliashar and MordechaiChevion (2014); “ $\mathrm{Zn} / \mathrm{Ga} \_\mathrm{DFO}$ iron-chelating complex attenuates the inflammatory process in a mouse model of asthma." Redox Biology2 814-81. DOI: 10.1016/j.redox.2014.06.009 PMid:25009783

\section{PMCid:PMC4085351}

[39] Heli H, Mirtorabi S and Karimian K. (2011); "Advances in iron chelation: an update."Expert OpinTher Pat. 2011 Jun;21(6):819-56.

DOI: $10.1517 / 13543776.2011 .569493$.

[40] Ikeda Yasumasa, IoriOzono, Soichiro Tajima, MizukiImao,YuyaHorinouchi, Yuki IzawaIshizawa, Yoshitaka Kihira, Licht Miyamoto, Keisuke Ishizawa, Koichiro Tsuchiya and Toshiaki Tamaki (2014). 'Iron Chelation by Deferoxamine Prevents Renal Interstitial Fibrosis in Mice with Unilateral Ureteral Obstruction'.PLOSONE Volume 9 Issue 2 e89355. DOI:10.1371/journal.pone.0089355

[41] Kang H, Yan Y, Jia P, Yang K, Guo C, Chen H, Qi J, Qian N, Xu X, Wang F, Li C, Guo L, Deng L(2016); "Desferrioxamine reduces ultrahigh-molecular-weight polyethyleneinduced osteolysis by restraining inflammatory osteoclastogenesis via heamoxygenase-1." Cell death \& disease ;7(10):e2435. DOI: 10.1038/cddis.2016.339.

[42] DemetriosVlahakos, NikolaosArkadopoulos, Georgia Kostopanagiotou, Sofia Siasiakou, LoukasKaklamanis, DimitriosDegiannis, Maria Demonakou, and VassiliosSmyrniotis (2012); "Deferoxamine Attenuates Lipid Peroxidation, Blocks Interleukin-6 Production, Ameliorates Sepsis Inflammatory Response Syndrome, and Confers Renoprotection After Acute Hepatic Ischemia in Pigs." Artif Organs, Vol. 36,No. 4. DOI:10.1111/j.1525-1594.2011.01385.x

[43] Zhang Xiao-Ying, Jiang-Bei Cao, Li-Ming Zhang, Yun-Feng $\mathrm{Li}$ and Wei-Dong $\mathrm{Mi}$ (2015); "Deferoxamine attenuates lipopolysaccharide induced neuroinflammation and memory impairment in mice." Journal of Neuroinflammation 12:20. DOI: $\underline{10.1186 / s 12974-015-0238-3}$ PMid:25644393 PMCid:PMC4323121

[44] Yanyan Li, Man Chen, YanyanXu, Xiao Yu, Ting Xiong, Min Du, Jian Sun, Liegang Li, Yuhan Tang and Ping Yao (2016); "Iron- 
Mediated Lysosomal Membrane

Permeabilization in Ethanol-Induced Hepatic Oxidative Damage and Apoptosis: Protective Effects of Quercetin." Oxidative Medicine and Cellular Longevity. Volume 2016, Article ID 4147610,15 pages.

DOI: $\underline{10.1155 / 2016 / 4147610}$

[45] Yury S. Tarahovsky, Yuri A. Kim, Elena A. Yagolnik, Eugeny N. Muzafarov (2014); "Flavonoid-membrane interactions: Involvement of flavonoid-metal complexes in raft signaling." Biochimica et Biophysica Acta (BBA) - Biomembranes Volume 1838, Issue 5, May 2014, Pages 1235-1246.

DOI:10.1016/j.bbamem.2014.01.021

[46] Chen, M.C., Ye, Y.Y., Ji, G. and Liu, J.W., (2010); "Hesperidin up-regulates hemeoxygenase-1 to attenuate hydrogen peroxide-induced cell damage in hepatic L02 cells". Journal of agricultural and food chemistry, 58 (6), pp. 3330- 3335.

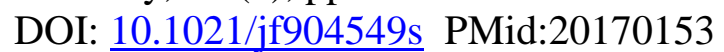

[47] Abraham NG, and Kappas A (2008); "Pharmacological and clinical aspects of heamoxygenase." Pharmacol Rev 2008; 60:79-127. DOI:10.1124/pr.107.07104
[48]NevenaMihailovicStanojevic,ZoranMiloradovi ,MilanIvanov,BrankBugarski,YurZicaJovoviT, Danijela Karanovi, Una-JovanaVaji, DraDenkaKomes and JelicaGruji-Milanovi (2016); "Upregulation of HemeOxygenase-1 in Response to Wild Thyme Treatment Protects against Hypertension and Oxidative Stress." Oxidative medicine and Cellular Longevity Volume 2, Article ID 1458793, 11 pages. DOI: $10.1155 / 2016 / 1458793$

[49] LilibethLanceta, Jacob M. Mattingly, Chi Li and John W. Eaton (2015)' How HemeOxygenase-1 Prevents Heme- Induced Cell Death'. PLoS ONE 10(8): e0134144. DOI:10.1371/journal.pone.0134144.

[50] Yuh-Mou Sue, Ching-Feng Cheng, ChihCheng Chang, Ying Chou, Cheng-HsienChen1 and Shu-Hui Juan (2009) 'Antioxidation and anti-inflammation by hea $m$ oxygenase-1 contribute to protection by tetramethylpyrazine against gentamicin-induced apoptosis in murine renal tubular cellsNephrol Dial Transplant 24: 769-777. DOI: 10.1093/ndt/gfn545. 